\title{
Tratamento da exacerbação da asma na criança com inaladores pressurizados de dose controlada e câmara expansora: dose segura e eficaz de salbutamol
}

Alexandra Sousa,* Helena Velho,* Pedro Almeida, ${ }^{* *}$ Teresa Maia Fernandes*

\section{RESUMO}

Objectivos: Os agonistas $\beta 2$ de curta duração são os fármacos de eleição no tratamento das exacerbações agudas de asma, sendo o salbutamol o mais frequentemente utilizado. No entanto, a dose recomendada é ainda controversa. O objectivo deste trabalho é determinar a dose segura e eficaz de salbutamol a administrar usando inaladores pressurizados de dose controlada e câmara expansora.

Fontes de dados: TRIP Database, National Guideline Clearinghouse, Guidelines Finder, The Cochrane Library, DARE Bandolier, MEDLINE.

Métodos de revisão: Pesquisa bibliográfica com os Medical Subject Headings, "Asthma/therapy" e "Albuterol/administration and dosage". Limitou-se a pesquisa a artigos publicados entre Janeiro de 2002 e Dezembro de 2012, em inglês, português, castelhano e francês. O nível de evidência e a força de recomendação foram atribuídos segundo a escala de Strenght of Recommendation Taxonomy da American Family Physician.

Resultados: Foram avaliados sete artigos: cinco normas de orientação clínica (NOC) e dois ensaios clínicos (EC). A dose recomendada pelas NOC varia entre duas e dez inalações ou puffs a cada 20 minutos. Cada puff equivale a uma inalação de 100 $\mu \mathrm{g}$ de salbutamol. Relativamente aos EC, as doses recomendadas são superiores às referidas nas NOC. Não foram relatados efeitos adversos significativos com as doses mencionadas.

Conclusões: Observa-se heterogeneidade nos diversos estudos, com NOC a sugerirem dois a quatro, dois a seis ou quatro a oito puffs. Os EC apontam para doses superiores, que podem atingir os 20 puffs por dose. Os autores consideram que existe segurança na administração de quatro a oito puffs por dose para o tratamento da exacerbação ligeira a moderada da asma em crianças (Força de Recomendação B). Cada dose não deverá ultrapassar os dez puffs (Força de Recomendação B).

Palavras-chave: Salbutamol; Exacerbação de Asma; Relação Dose-Resposta; Eficácia; Segurança.

\section{INTRODUÇÃO}

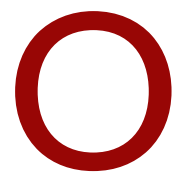

objectivo do tratamento da asma é atingir e manter o seu controlo clínico. A terapêutica inalatória é a base do tratamento pelas suas características de eficácia e segurança. Os agonistas $\beta 2$ de rápida acção constituem o tratamento de primeira linha nas crises de asma, sendo o salbutamol o fármaco mais usado no nosso país. ${ }^{1}$

*Internas Complementares Medicina Geral e Familiar, ULS Matosinhos

**Interno Complementar Medicina Geral e Familiar, ACES Maia
O método habitual para administração da terapêutica inalatória de alívio no Serviço de Urgência (SU) tem sido a nebulização, ${ }^{1}$ no entanto são várias as desvantagens apontadas ao uso dos nebulizadores, nomeadamente o facto de serem pouco portáteis, ser necessária uma fonte de energia e de gás pressurizado, ser um tratamento demorado, ser necessária a limpeza do aparelho, haver risco de contaminação, existir variabilidade com diferentes aparelhos, ser dispendioso, entre outras. $^{2}$ Comparativamente, a utilização de inaladores 
pressurizados de dose controlada (MDI) com câmara expansora tem diversas vantagens, nomeadamente a curta duração do tratamento, a menor possibilidade de contaminação e a elevada reprodutibilidade das doses. ${ }^{2,3}$ Vários estudos demonstraram a boa relação custo-eficácia da utilização de MDI com câmara expansora nas crises leves a moderadas de asma em crianças. Ploin e colaboradores ${ }^{4}$ efectuaram um estudo comparativo entre administração de salbutamol através de MDI com câmara expansora e através de nebulizador, concluindo que a eficácia dos dois tratamentos foi semelhante, apontando uma melhor tolerabilidade nos casos que utilizaram MDI com câmara expansora. Assim, de acordo com este e outros estudos semelhantes, a aerossoloterapia com salbutamol através de MDI com câmara expansora passou a ser o método de escolha segundo as principais Normas de Orientação Clínica (NOC) nacionais ${ }^{5}$ e internacionais, ${ }^{6-10}$ sendo o uso dos nebulizadores feito apenas a título excepcional e devidamente fundamentado.

Porém, não é consensual a equivalência de doses entre o salbutamol nebulizado e o administrado mediante MDI com câmara expansora. A dose mais habitual de salbutamol nebulizado é de 0,15 a $0,2 \mathrm{mg} / \mathrm{kg} /$ dose e diversos estudos defendem proporções muito díspares entre MDI e nebulização, com valores que vão dos 1:2 a 1:10 (MDI:nebulização). ${ }^{4,11-16}$

A dose de salbutamol recomendada no resumo das características do medicamento, de 100 a $200 \mu \mathrm{g}$ (um a dois puffs), poderá ser inadequada, uma vez que as actualizações das NOC internacionais apresentam recomendações com doses superiores. Contudo, parece haver alguma relutância por parte da classe médica em utilizar doses superiores, talvez por receio dos possíveis efeitos secundários, como taquicardia, trémulo, cefaleias, hipocaliémia, entre outros. ${ }^{17-19}$

Por outro lado, a exposição sistémica em indivíduos jovens pode ser menor do que a esperada por estes apresentarem uma deposição do fármaco nas vias aéreas superiores e uma clearance pulmonar aumentadas. Assim, as crianças mais novas parecem estar protegidas contra a exposição sistémica excessiva. A prática comum de reduzir a dose em crianças mais jovens com medo de exposição sistémica excessiva pode, desta forma, acarretar o uso de doses possivelmente sub-terapêuticas. ${ }^{20}$
Neste sentido, os autores propõem-se com este trabalho determinar a dose segura e eficaz de salbutamol a utilizar por MDI com câmara expansora no tratamento de crianças com exacerbações ligeiras a moderadas de asma.

\section{METODOLOGIA}

Realizou-se uma pesquisa de NOC, meta-análises, artigos de revisão e estudos originais, na Pubmed e em bases de dados online de Medicina Baseada na Evidência (TRIP Database, National Guideline Clearinghouse, Guidelines Finder, The Cochrane Library, DARE, Bandolier, MEDLINE), Índex de Revistas Médicas Portuguesas e referências bibliográficas dos artigos seleccionados. Para a pesquisa foram utilizados os termos Medical Subject Headings (MeSH), Asthma/therapye Albuterol/administration and dosage, ou as palavras-chave Asthma e Albuterol nas bases de dados que não suportam pesquisa por termos MeSH. No Índex de Revistas Médicas Portuguesas a pesquisa foi realizada com os termos Asma e Salbutamol.

Foram pesquisados artigos publicados entre Janeiro de 2002 e Dezembro de 2012, em Inglês, Português, Castelhano e Francês.

Foram definidos os seguintes critérios de inclusão:

- População: crianças até aos 18 anos com diagnóstico de asma e com exacerbações ligeiras a moderadas;

- Intervenção: salbutamol por MDI com câmara expansora com doses acima do preconizado, isto é superiores a um ou dois puffs;

- Comparação: salbutamol por MDI com câmara expansora nas doses preconizadas, um a dois puffs;

- Outcome: melhoria clínica e ausência de efeitos laterais significativos.

Foram excluídas as crianças sem diagnóstico de asma prévio e as que se encontravam com exacerbações graves.

Para avaliação da qualidade dos estudos e atribuição da força de recomendação foi utilizada a escala Strenght of Recommendation Taxonomy (SORT) da American Family Physician (quadro I).

\section{RESULTADOS}

Foram encontrados 425 artigos, dos quais 108 artigos no TRIP Database, 16 na National Guideline Clea- 


\begin{tabular}{|c|c|c|}
\hline & Classificação & Significado \\
\hline \multirow[t]{3}{*}{ Força de Recomendação } & A & $\begin{array}{l}\text { Recomendação baseada em evidência consistente, } \\
\text { de boa qualidade, orientada para o doente }\end{array}$ \\
\hline & B & $\begin{array}{l}\text { Recomendação baseada em evidência inconsistente, } \\
\text { de qualidade limitada, orientada para o doente }\end{array}$ \\
\hline & C & $\begin{array}{l}\text { Recomendação baseada em consensos, prática } \\
\text { clínica, opinião, orientada para a doença ou séries de } \\
\text { casos acerca do diagnóstico, tratamento prevenção } \\
\text { ou rastreio }\end{array}$ \\
\hline \multirow[t]{3}{*}{ Níveis de Evidência } & 1 & $\begin{array}{l}\text { Estudos de boa qualidade, evidência orientada para } \\
\text { o doente }\end{array}$ \\
\hline & 2 & $\begin{array}{l}\text { Estudos de qualidade limitada, evidência orientada } \\
\text { para o doente }\end{array}$ \\
\hline & 3 & Outra evidência \\
\hline
\end{tabular}

dois a quatro $p u f f s$ a cada 20 min, até um máximo de dez puffs por dose, com força de recomendação C, uma vez que não especifica que estudos lhe deram origem.

A recomendação emitida em 2009 pelo $D e$ partment of Veteran Affairs, "Va/DoD", preconiza uma dose de dois a seis puffs a cada 20 minutos, para crianças com seis ou mais anos de idade. Mais uma vez não é especificada a força de recomendação relativa à dose de sal-

ringhouse, 19 no The Cochrane Library, 53 no DARE, 211 na Pubmed e 18 artigos no Índex de Revistas Médicas Portuguesas. Foram excluídos os artigos repetidos, os não concordantes com os objectivos ou os que não cumpriam os critérios de inclusão, o que resultou numa selecção de 53 artigos para leitura.

Destes, foram seleccionados sete artigos para inclusão nesta revisão: cinco NOC e dois EC, porque os restantes 46 artigos lidos não preenchiam totalmente os critérios de inclusão.

\section{NORMAS DE ORIENTAÇÃO CLÍNICA}

As forças de recomendação atribuídas pelas cinco NOC analisadas foram convertidas para a taxonomia SORT.

A dose de salbutamol recomendada pelo GINA é de dois a quatro $p u f f s$ a cada 20 minutos na primeira hora, seguida de repetições de dois a quatro puffs a cada 3-4 horas, na crise ligeira, ou seis a dez puffs a cada 1-2 horas, na crise moderada. Embora seja explicada a metodologia utilizada na elaboração desta NOC, no que diz respeito à dose de salbutamol, não é explícita a força de recomendação ou os estudos em que se baseia, pelo que os autores atribuíram uma força de recomendação $\mathrm{C}$, correspondente a um consenso de peritos.

A British Thoracic Society $(B T S)^{8}$ emitiu uma NOC para o tratamento da asma em crianças com idade superior a dois anos. Os autores recomendam o uso de butamol a utilizar, pelo que os autores atribuem uma força de recomendação $\mathrm{C}$ a esta orientação.

O National Medical Research Council (Singapore Ministry of Health ${ }^{9}$ recomenda uma dose de quatro a oito puffs ou 0,2-0,3 puffs $/ \mathrm{kg}$ a cada 20 minutos (com um máximo de 10 puffs/dose), com uma força de recomendação A, que é compatível com a força de recomendação A da escala SORT, uma vez que esta sociedade afirma basear esta recomendação em estudos de elevada qualidade (meta-análises, revisões sistemáticas de ensaios clínicos controlados e aleatorizados e ensaios clínicos controlados e aleatorizados).

Em 2007, o National Heart, Lung, and Blood Institu$t e^{10}$ emitiu a recomendação de utilização de quatro a oito puffs a cada $20 \mathrm{~min}$ na primeira hora. Os autores atribuíram uma força de recomendação $\mathrm{C}$ uma vez que não é clara a sustentação científica utilizada.

\section{ENSAIOS CLÍNICOS}

Bønnelykke et a ${ }^{20}$ realizaram um ensaio clínico não aleatorizado com 65 crianças, dos três aos dezasseis anos, com asma leve a moderada. Todas as crianças fizeram uma inalação de quatro puffs de salbutamol através de um MDI com câmara expansora, independentemente da idade. Foi medido o valor sérico de salbutamol 20 minutos após a inalação constatando-se que, para doses semelhantes, a exposição sistémica é menor nas crianças mais novas. Concluíram então que a dose 
de salbutamol administrada através de MDI com câmara expansora deve ser ajustada à idade ou tamanho e não apenas ao peso pois tal causa redução da exposição em crianças mais novas com o risco correspondente de dosagem sub-óptima. Não foram relatados quaisquer efeitos laterais no estudo. Como limitações, este estudo apresenta uma "disease oriented evidence» (DOE), pois avalia o valor sérico de salbutamol. No entanto, foi incluído na revisão, uma vez que um dos outcomes avaliados pelos autores é a ausência de efeitos laterais significativos com doses mais elevadas de salbutamol do que as preconizadas. Trata-se de um ensaio clínico não aleatorizado, com uma amostra reduzida, pelo que se atribuiu um nível de evidência 3.

Fernández et a ${ }^{21}$ realizou um ensaio clínico aleatorizado com dupla ocultação, que incluiu 324 crianças entre os 2 e os 14 anos, com diagnóstico prévio de asma, e que se dirigiram ao SU por uma exacerbação. Estabeleceram-se dois grupos de tratamento: um grupo de crianças recebeu um número de puffs equivalente a metade do seu peso e o outro grupo recebeu um número de puffs equivalente a um terço do seu peso (mínimo de cinco puffs e máximo de vinte puffs por dose administrada). As doses foram repetidas a cada $20 \mathrm{mi}-$ nutos até três vezes. Os efeitos avaliados foram o valor do Pulmonary Score, que consiste numa escala validada para avaliação de gravidade e tem como base critérios clínicos (frequência respiratória, alterações auscultatórias e uso de músculos acessórios da respiração) e a saturação de oxigénio por oximetria de pulso. Não se encontraram diferenças estatisticamente significativas em nenhum dos outcomes avaliados entre os dois grupos. Não foram descritos efeitos laterais significativos para ambas as doses. Tratase de um ensaio clínico com bom desenho de estudo cuja amostra apresentava as mesmas características em ambos os grupos, tanto à chegada ao SU como após o tratamento. O estudo avaliou out- comes clínicos através de uma escala validada, sendo classificada com Nível de Evidência 1. De salientar que a dose mínima utilizada neste estudo foi de cinco puffs, sendo mais elevada do que as preconizadas nas NOC emitidas pela British Thoracic Societye GINA. Ver resumo dos resultados no Quadro III.

\section{DISCUSSÃO/CONCLUSÃO}

Os estudos publicados até à data apontam para uma utilização de dois a oito $p u f f s$ a cada 20 min na primeira hora. Esta heterogeneidade observa-se nos diversos estudos, com as NOC a sugerirem dois a quatro, dois a seis ou quatro a oito puffs. Os ensaios clínicos apontam para doses superiores, podendo atingir os 20 puffs por dose. É de realçar que os estudos de maior qualidade recomendam doses mais elevadas de salbutamol, mínimo de quatro a oito puffs.

Assim, os autores desta revisão consideram que existe segurança na administração de quatro a oito puffs por dose para o tratamento da exacerbação ligeira a moderada de asma em idade pediátrica com Força de Recomendação B. Cada dose não deverá ultrapassar os 10 puffs (Força de Recomendação B). Em relação à regularidade das tomas, na primeira hora está descrita em todos os artigos a repetição a cada 20 minutos da mesma dose, período após o qual deverá ser administrado em intervalos de 1-4h.

Em relação aos efeitos adversos, todos os ensaios clí-

\begin{tabular}{|c|c|c|c|}
\hline Entidade & Ano & Dose Recomendada & FR \\
\hline $\begin{array}{l}\text { Global Initiative for Asthma } \\
\text { (GINA) }\end{array}$ & 2011 & $\begin{array}{l}\text { 2-4 puffs cada } 20 \text { min (1. hora) } \\
\text { 2-4 puffs cada } 3-4 \text { horas - Crise ligeira } \\
\text { 6-10 puffs a cada } 1-2 \text { horas - Crise moderada }\end{array}$ & $\mathrm{C}$ \\
\hline British Thoracic Society & 2011 & $\begin{array}{l}\text { Crianças com idade }>2 \text { anos } \\
2-4 \text { puffs cada } 10-20 \text { min (máx. } 10 \text { puffs/dose) }\end{array}$ & $\mathrm{C}$ \\
\hline VA/DoD & 2009 & $\begin{array}{l}\text { Crianças com idade } \geq 6 \text { anos } \\
2-6 \text { puffs cada } 20 \mathrm{~min}\end{array}$ & $\mathrm{C}$ \\
\hline Singapore Ministry of Health & 2008 & $\begin{array}{l}\text { 4-8 puffs ou } 0.2-0.3 \text { puffs } / \mathrm{kg} \text { cada } 20 \mathrm{~min} \\
\text { Máx } 10 \text { puffs/dose }\end{array}$ & $A$ \\
\hline $\begin{array}{l}\text { National Heart, Lung, } \\
\text { and Blood Institute }\end{array}$ & 2007 & $\begin{array}{l}\text { 4-8 puffs cada } 20 \text { min ( } 1 .^{\text {a hora) }} \\
\text { 4-8 puffs cada } 1-4 \text { horas }\end{array}$ & $\mathrm{C}$ \\
\hline
\end{tabular}

FR: Força de Recomendação 


\begin{tabular}{|c|c|c|c|c|}
\hline Referência & $\begin{array}{l}\text { Tipo de Estudo/ } \\
\text { /População }\end{array}$ & Metodologia & Resultados/Conclusões & NE \\
\hline $\begin{array}{l}\text { J. Benito Fernández } \\
\text { et al, Anales de } \\
\text { Pediatría, } 2006\end{array}$ & $\begin{array}{l}\text { ECA com dupla } \\
\text { ocultação } \\
\text { crianças } 2-14 \\
\text { anos }(n=324)\end{array}$ & 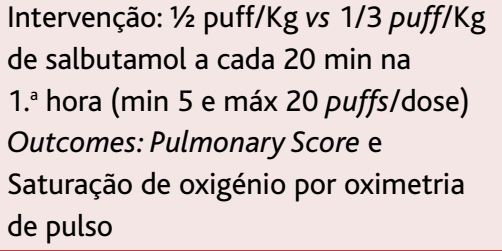 & $\begin{array}{l}\text { Não relatados efeitos laterais } \\
\text { significativos } \\
\text { Eficácia semelhante para ambas as } \\
\text { doses ( } 1 / 2 \text { puff } / \mathrm{kg} \text { ou } 1 / 3 \text { puff } / \mathrm{kg} \text { ) }\end{array}$ & 1 \\
\hline $\begin{array}{l}\text { Bønnelykke et al, } \\
\text { Br Journal of } \\
\text { Pharmacology, } \\
2007\end{array}$ & $\begin{array}{l}\text { EC não } \\
\text { aleatorizado } \\
\text { crianças dos } 3 \\
\text { aos } 16 \text { anos } \\
(n=65)\end{array}$ & $\begin{array}{l}\text { Intervenção: } 4 \text { puffs de salbutamol por } \\
\text { MDI a todas crianças } \\
\text { Outcome: valor sérico de salbutamol } \\
20 \text { min após administração }\end{array}$ & $\begin{array}{l}\text { Sem relato de quaisquer efeitos laterais } \\
\text { Com a mesma dose: exposição sistémica } \\
\text { é menor nas crianças mais novas } \\
\text { A dose deve ser ajustada à idade ou } \\
\text { tamanho e não ser fixa nos puffs } / \mathrm{kg}\end{array}$ & 3 \\
\hline
\end{tabular}

NE: Nível de Evidência; ECA: Ensaio Clínico Aleatorizado; EC: Ensaio Clínico

nicos apresentaram ausência de efeitos laterais significativos com as doses instituídas.

Os estudos analisados possuem algumas limitações, nomeadamente escassez de estudos direccionados para a avaliação da dose mínima eficaz de salbutamol, curta duração e desenhos de estudo heterogéneos.

São necessários mais estudos controlados e aleatorizados, de longa duração e de elevada qualidade, sobre a determinação da dose e intervalo de repetição após a primeira hora assim como a dose máxima por dia. Carece também de estudo a determinação da dose a utilizar em crianças com menos de dois anos, visto nestas ser provavelmente necessária uma maior dose $/ \mathrm{kg}$ devido à fisiologia inerente a esta faixa etária, o que acarreta o risco de dosagem sub-terapêutica. A área dos medicamentos administrados a crianças levanta questões éticas, o que poderá contribuir para a escassez de bibliografia.

\section{REFERÊNCIAS BIBLIOGRÁFICAS}

1. Fernandez JB. Tratamiento con broncodilatadores en urgencias de pediatría: nebulización versus inhalación con cámara espaciadora. Arch Pediatr Urug 2003 Ago;74 (2):114-8.

2. Dolovich MB, Ahrens RC, Hess DR, Anderson P, Dhand R, Rau JL, et al. Device selection and outcomes of aerosol therapy: Evidence-based guidelines: American College of Chest Physicians/American College of Asthma, Allergy, and Immunology. Chest 2005 Jan; 127 (1): 335-71.

3. AARC Clinical Practice Guideline. Selection of an aerosol delivery for neonatal and pediatric patients. Respir Care 1995 Dec; 40 (12): 1325-
35.

4. Ploin D, Chapuis FR, Stamm D, Robert J, David L, PG, et al. C. High-dose albuterol by metered dose inhaler plus a spacer device versus nebulization in preschool children with recurrent wheezing: a double-blind, randomized equivalence trial. Pediatrics 2000 Aug; 106 (2 Pt 1): 3117.

5. Direcção-Geral de Saúde. Norma 021/2011 - Cuidados Respiratórios Domiciliários: Prescrição de Aerossolterapia. Lisboa: DGS; 2011.

6. From the Global Strategy for Asthma Management and Prevention, Global Initiative for Asthma (GINA) 2012. Disponível em: http://www. ginasthma.org/ [acedido em 01/03/2013].

7. Management of Asthma Working Group. VA/DoD clinical practice guideline for management of asthma in children and adults. Washington (DC): Department of Veteran Affairs, Department of Defense; 2009. p. 126.

8. British Thoracic Society, Scottish Intercollegiate Guidelines Network. British Guideline on the Management of Asthma - A national clinical guideline. May 2008, Revised January 2012. Disponível em: http://www.sign.ac.uk/pdf/sign101.pdf [acedido em 01/03/2013].

9. Singapore Ministry of Health. Management of asthma. Singapore: Singapore Ministry of Health; 2008. p. 80.

10. National Heart Lung and Blood Institute. National Asthma Education and Prevention Program. Expert Panel Report 3: Guidelines for the Diagnosis and Management of asthma. Washington, DC: US Department of Health and Human Services; 2007.

11. Leversha AM, Campanella SG, Aickin RP, Asher MI. Costs and effectiveness of spacer versus nebulizer in young children with moderate and severe acute asthma. J Pediatr 2000 Apr; 136 (4): 497-502.

12. CCMRC/GINA. Workshop on asthma management and prevention in the Caribbean. July 1-3, 1997, Trinidad. Commonwealth Caribbean Medical Research Council. Global Initiative for Asthma. West Indian Med J 1998 Dec;47 (4): 133-52. 
13. Delgado A, Chou K, Silver EJ, Crain E. Nebulizers versus metered-dose inhalers with spacers for bronchodilator therapy to treat wheezing in children aged 2 to 24 months in a pediatric emergency department. Arch Pediatr Adolesc Med 2003 Jan; 157 (1): 76-80.

14. Osmond M, Diner B. Nebulizers versus inhalers with spacers for acute asthma in pediatrics. Ann Emerg Med 2004 Mar; 43 (3): 413-5.

15. Benito Fernández J, González Balenciaga M, Capapé Zache S, Vázquez Ronco M, Mintegi Raso S. Salbutamol via metered-dose inhaler with spacer versus nebulization for acute treatment of pediatric asthma in the emergency department. Pediatr Emerg Care 2004 Oct; 20 (10): 6569.

16. Rubilar L, Castro-Rodríguez JA, Girardi G. Randomized trial of salbutamol via metered-dose inhaler with spacer versus nebulizer for acute wheezing in children less than 2 years or age. Pediatr Pulmonol 2000 Apr; 29 (4): 264-9.

17. Hardman JG, Limbird LE, Gilman AG. Goodman \& Gilman's The Pharmacological Basis of Therapeutics. 10th ed. New York: McGraw-Hill; 2001.

18. Ministério da Saúde, Infarmed. Prontuário Terapêutico Online. Disponível em: http://www.infarmed.pt/prontuario/index.php [acedido em 01/03/2013].
19. Albuterol (salbutamol): Drug information. UpToDate. Waltman, MA:UpToDate; 2013.

20. Bønnelykke K, Jespersen JJ, Bisgaard H. Age dependent systemic exposure to inhaled salbutamol. Br J Clin Pharmacol 2007 Aug; 64 (2): 2414.

21. Benito Fernández J, Trebolazabala Quirante N, Landa Garriz M, Mintegi Raso S, González Díaz C. Broncodilatadores inhalados mediante MDI con cámara espaciadora en urgencias pediátricas: ¿cuál es la dosis? An Pediatr (Barc) 2006 Ene; 64 (1): 46-51.

\section{CONFLITOS DE INTERESSE}

Os autores declaram não ter conflito de interesses.

\section{ENDEREÇO PARA CORRESPONDÊNCIA}

Alexandra Sousa

Travessa Francisco Sá Carneiro, 324 - $1^{\circ}$ Dto

4450-677 Leça da Palmeira

E-mail: sousalexandra@gmail.com

Recebido em 25/04/2012

Aceite para publicação em 08/03/2013

\section{ABSTRACT}

\section{TREATMENT OF ACUTE ASTHMA EXACERBATIONS IN CHILDREN WITH A PRESSURIZED METERED DOSE INHALER} AND SPACER: SAFE AND EFFECTIVE DOSING OF SALBUTAMOL

Aim and Objectives: Inhaled short-acting beta 2-agonists are the mainstay of treatment of acute asthma exacerbations. Salbutamol (also known as albuterol) is the most frequently used of these drugs in acute settings, however the recommended dose is still the subject of debate. The aim of this study is to determine the safe and effective dose of salbutamol delivered by a metered-dose inhaler with a spacer.

Data sources: TRIP Database, National Guideline Clearinghouse, Guidelines Finder, The Cochrane Library, DARE, Bandolier, MEDLINE.

Methods of the review: bibliographic search using the MeSH terms: «Asthma/therapy» and «Albuterol/administration and dosage». The search was limited to articles published between January 2001 and 2011 in English, Spanish, French and Portuguese. The Strength of Recommendation Taxonomy of the American Family Physician Journal was used to assess the Level of Evidence. Results: Seven articles were evaluated including five guidelines and two clinical trials. The dose recommended by the guidelines was between two and ten puffs every 20 minutes. Each puff is equivalent to $100 \mu \mathrm{g}$ of salbutamol by inhalation. In the clinical trials, the recommended doses are higher to those referred to by the guidelines. Adverse effects were not reported with these doses.

Conclusions: There is some heterogeneity of the included studies. Some guidelines recommend two to four puffs, others two to six puffs, and others four to eight puffs at a time. The clinical trials report on higher doses, up 20 puffs per dose. The authors consider that it is safe to administer four to eight puffs per dose in the treatment of a child with a mild to moderate exacerbation of asthma (Strength of Recommendation B). Each dose should not be more than ten puffs (Strength of recommendation B).

Keywords: Albuterol; Asthma Exacerbation; Dose Response Relationship; Efficacy; Safety. 